\title{
Self-assembly of silicide quantum dot arrays on stepped silicon surfaces by reactive epitaxy
}

Cite as: Appl. Phys. Lett. 91, 263106 (2007); https://doi.org/10.1063/1.2828036

Submitted: 12 November 2007 . Accepted: 03 December 2007 . Published Online: 27 December 2007

L. Fernández, M. Löffler, J. Cordón, and J. E. Ortega

ARTICLES YOU MAY BE INTERESTED IN

Self-organized growth of high density magnetic Co nanodot arrays on a Moiré template Applied Physics Letters 96, 013107 (2010); https://doi.org/10.1063/1.3280900

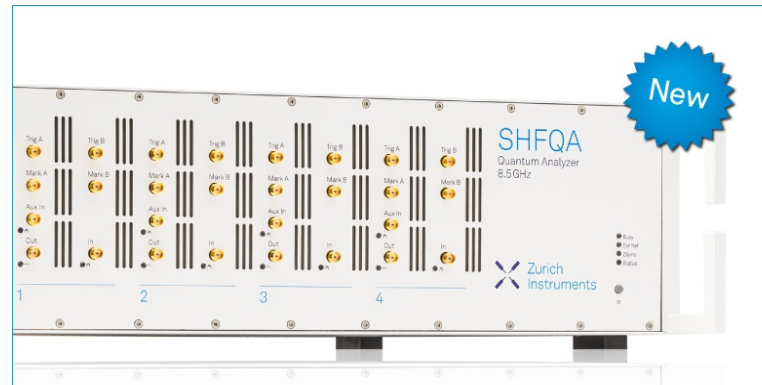

\section{Your Qubits. Measured.}

Meet the next generation of quantum analyzers

- Readout for up to 64 qubits

- Operation at up to $8.5 \mathrm{GHz}$.

Find out more

- Signal optimization with minimal latency 


\title{
Self-assembly of silicide quantum dot arrays on stepped silicon surfaces by reactive epitaxy
}

\author{
L. Fernández ${ }^{a}$ \\ Departamento de Física Aplicada I, Universidad del País Vasco, Plaza de Oñate 2, E-20018 Donostia-San \\ Sebastián, Spain \\ M. Löffler \\ Lehrstuhl für Festkörperphysik, Universität Erlangen, Staudtstrasse 7, D-91058 Erlangen, Germany \\ J. Cordón and J. E. Ortega \\ Departamento de Física Aplicada I, Universidad del País Vasco, Plaza de Oñate 2, E-20018 Donostia-San \\ Sebastián, Spain and DIPC and Unidad de Física de Materiales CSIC/UPV, Manuel Lardizábal 4. \\ E-20018 Donostia-San Sebastián, Spain
}

(Received 12 November 2007; accepted 3 December 2007; published online 27 December 2007)

\begin{abstract}
Reactive epitaxy of Co on vicinal $\mathrm{Si}(111)$ surfaces is found to be a flexible and a convenient method for the preparation of dense arrays of Co silicide quantum dots. In the present work, submonolayer amounts of Co were deposited at $800 \mathrm{~K}$ on vicinal and flat $\mathrm{Si}$ surfaces, analyzing the resulting structures by scanning tunneling microscopy. On vicinal $\mathrm{Si}(111)$ surfaces with relatively narrow $(\sim 40-100 \AA)$ terrace width, such reactive epitaxy leads to self-assembled arrays of $\mathrm{CoSi}_{2}$ quantum dots with a sharp size distribution function. In contrast, the growth of Co on flat $\mathrm{Si}(111)$ results in an inhomogeneous array of dots mixed with a variety of silicide phases. (c) 2007 American Institute of Physics. [DOI: 10.1063/1.2828036]
\end{abstract}

Self-assembly is revealed as the straightforward alternative to obtain ordered arrays of nanoscale units for nanotechnology applications. In this context, the vicinal surface approach appears as ideal for the preparation and tailoring of self-assembled epitaxial nanostructures. Ordered arrays of nanodots or nanowires have been already successfully designed using metallic or semiconducting vicinal surfaces as substrates. Remarkable is the growth of metallic dots of $\mathrm{Ag}$ (Ref. 1) or Co (Ref. 2) on vicinal $\mathrm{Au}(111)$, the latter exhibiting magnetic behavior. Concerning the preparation of nanowires, interesting avenues have been already explored toward lateral nanostructuration of rare earth silicides, such as $\mathrm{Gd}$, $\mathrm{Yb}$, or Dy silicides, on vicinal silicon. ${ }^{3-5}$ In contrast, very little has been investigated on the epitaxy and selforganization of laterally nanostructured transition metal (TM) silicide structures, despite their technological importance.

TMs are known to react strongly with silicon producing silicides that in most of the cases render intermetallic compounds, which are good electrical conductors with resistivity comparable to metals. The good conductivity and chemical stability of TMs have motivated their implementation in silicon integrated circuits, with applications as low resistivity interconnects or contacts. Among the wide variety of TMs compounds, Co disilicides are particularly attractive. They exhibit thermal stability to temperatures as high as $1200 \mathrm{~K}$, sharp interface, and low contact resistance with silicon, leading to stable and reliable Schottky or Ohmic contacts. ${ }^{6}$ In this work, we study the reactive epitaxy (RE) of Co on vicinal $\mathrm{Si}(111)$ surfaces by deposition of low amounts of Co in the submonolayer range. $\mathrm{RE}$ of $\mathrm{Co}$ on $\mathrm{Si}$ involves strongly exothermic reactions of both materials and radical rearrangements of $\mathrm{Co}$ and $\mathrm{Si}$ atoms. ${ }^{7}$ In the submonolayer coverage regime, such reactive growth results in a rich phase diagram

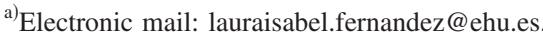

of $\mathrm{Co} / \mathrm{Si}(111)$ with a variety of superlattice reconstructions and stoichiometries that can coexist. In this work, we show that the growth of $\mathrm{CoSi}_{2}$ can be improved with the use of stepped surfaces, since these filter out undesired silicide phases during RE at low temperature. The use of stepped $\mathrm{Si}(111)$ as a template to nucleate disilicide nanoislands was already tested using surfaces with terrace sizes varying from 250 to $700 \AA$ and solid phase epitaxy. ${ }^{8}$ It has been claimed that terraces in the 250-400 $\AA$ range have an adverse effect on the adatom diffusion, acting against shape transition and formation of flattop silicide islands. In contrast to this conclusion, here we demonstrate that the stabilization of flattop $\mathrm{CoSi}_{2}$ nanoislands with controllable size and density can be actually achieved during RE of Co and using vicinal $\mathrm{Si}(111)$ surfaces with relatively narrow $(\sim 40-100 \AA)$ terraces.

Experiments have been carried out in situ in an ultrahigh vacuum system with a base pressure of $7 \times 10^{-11}$ mbar. The analysis chamber is equipped with an Omicron variable temperature scanning tunnel microscope (STM) and low-energy electron diffraction (LEED) optics. Flat and vicinal Si(111) surfaces with $4.6^{\circ}$ of miscut were used as substrates. Samples were prepared by a thorough degassing at $800 \mathrm{~K}$ for several hours in vacuum followed by heating a few times to around $1500 \mathrm{~K}$. Flashes were limited to $10 \mathrm{~s}$ in order to prevent the pressure to reach values higher than $6 \times 10^{-10}$ mbar. The samples were then rapidly cooled to $1220 \mathrm{~K}$ and then slowly down to $1000 \mathrm{~K}$. A final annealing cycle at $1000 \mathrm{~K}$ for $10 \mathrm{~min}$ improved the surface quality. LEED and STM measurements were routinely carried out after this process. Flat surfaces were characterized by fewmicron-size terraces with the $(7 \times 7)$ reconstruction, whereas stepped surfaces revealed terraces fully $(7 \times 7)$ reconstructed with steps that could be both bilayer $(0.31 \mathrm{~nm})$ or threebilayer $(0.93 \mathrm{~nm})$ high. Therefore, the terrace width was found to vary between 40 and $100 \AA$. Co was evaporated in submonolayer ranges $(0.2-0.4 \mathrm{ML})$ at a substrate tempera- 

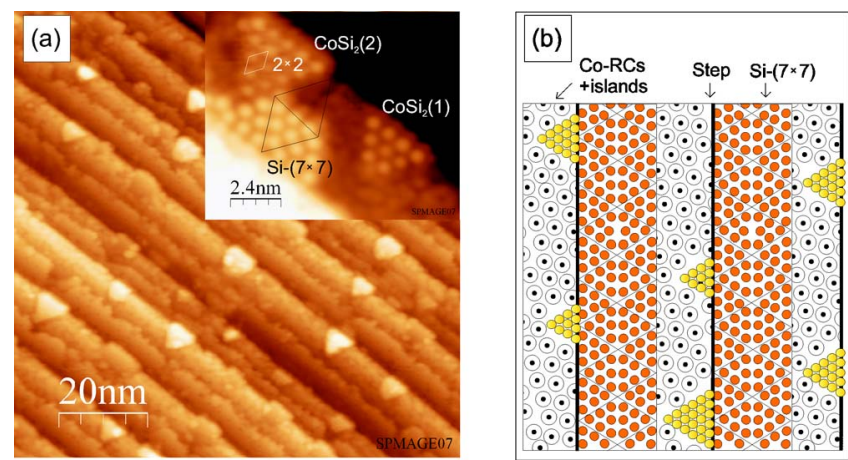

FIG. 1. (Color online) Growth of 0.2 ML of Co on vicinal Si(111) surface. (a) Empty-state STM topography image $\left(V_{\text {tip }}\right.$ is $\left.+2.1 \mathrm{~V}\right)$ that discloses triangular $\mathrm{CoSi}_{2}$ islands and $\mathrm{Co} \mathrm{RCs}$ on the step terraces. There are clean $\mathrm{Si}(111)-(7 \times 7)$ patches that appear at a different level. The inset reveals the flattop of the $\mathrm{CoSi}_{2}$ islands that exhibit a $2 \times 2$ surface reconstruction. (b) Schematic description of the early growth stage of Co that show the nucleation position of the islands and Co RCs which starts to nucleate at the upper side of the step, on the half unit cell of the $\mathrm{Si}(111)-(7 \times 7)$. Co RCs would nucleate around the islands, saturating the terrace in the proximity of the step edge. The uncoved parts of the surface maintain the $\mathrm{Si}(111)-(7$ $\times 7)$ surface reconstruction up to the lower step edge.

ture of $800 \mathrm{~K}$ with deposition rates varying from 0.2 to $0.3 \mathrm{ML} / \mathrm{min}$. After deposition, the samples were immediately cooled down to $300 \mathrm{~K}$ for measurements.

Figure 1(a) shows the early growth stage of Co on vicinal Si(111) and Fig. 1(b) schematically summarizes the STM observations. The deposition of $0.2 \mathrm{ML}$ leads to the formation of two-dimensional triangular islands that grow from the upper step edge and inside the terrace. They are $2 \AA$ high and exhibit atomically flattops with a $2 \times 2$ surface reconstruction, which is a characteristic of $\mathrm{CoSi}_{2}$ clusters grown during reactive epitaxy. ${ }^{9}$ The inset of Fig. 1(a) reveals the way the disilicide quantum dots begin to nucleate. They prefer the half unit cell (HUC) of the $\mathrm{Si}(111)-(7 \times 7)$ that is closer to the step edge, adopting the threefold symmetry of the $\mathrm{Si}(111)-(7 \times 7)$ (island 1$)$. Furthermore, there is a preferential nucleation on unfaulted HUCs, as observed during the homoepitaxy of $\mathrm{Si}$ on $\mathrm{Si}(111) .{ }^{10}$ The island marked as 2 shows that these grow laterally by extending to neighboring HUCs of the $\mathrm{Si}(111)-(7 \times 7)$, although they keep their triangular shape. Additionally, a different silicide phase, namely, the Co ring cluster (Co RC) phase, is detected at the same terrace level. RCs are known to be formed in all TMs. With $\mathrm{Co}$, they appear at very low coverage saturating the surface above 0.12/0.14 ML with a characteristic $\sqrt{7} \times \sqrt{7}$ superstructure. ${ }^{11}$ Both islands and Co RCs are the only silicide structures found on this sample. The rest of the surface exhibits clean $\mathrm{Si}(111)-(7 \times 7)$ patches that appear at a different level and close to the lower side of the step.

A denser array of Co silicide quantum dots $\left(8 \times 10^{-4} \mathrm{~nm}^{-2}\right)$ is formed by increasing the Co coverage to 0.4 ML, as shown in Fig. 2(a). In the close view of Fig. 2(b), we note the change in size and island geometry with respect to the early nucleation stage of $0.2 \mathrm{ML}$. In the $0.4 \mathrm{ML}$ array, the islands present diameters of around $15 \mathrm{~nm}$, often showing growth over several steps. They are $2 \mathrm{~nm}$ height and have flat tops and $2 \times 2$ surface reconstruction, namely, the characteristic $\mathrm{CoSi}_{2}$ features. In Fig. 2(c), we analyze the island size distribution shown in Fig. 2(a) by considering their perimeter. ${ }^{12}$ The island perimeter distribution can be fitted to a Gaussian function with maximum centered at
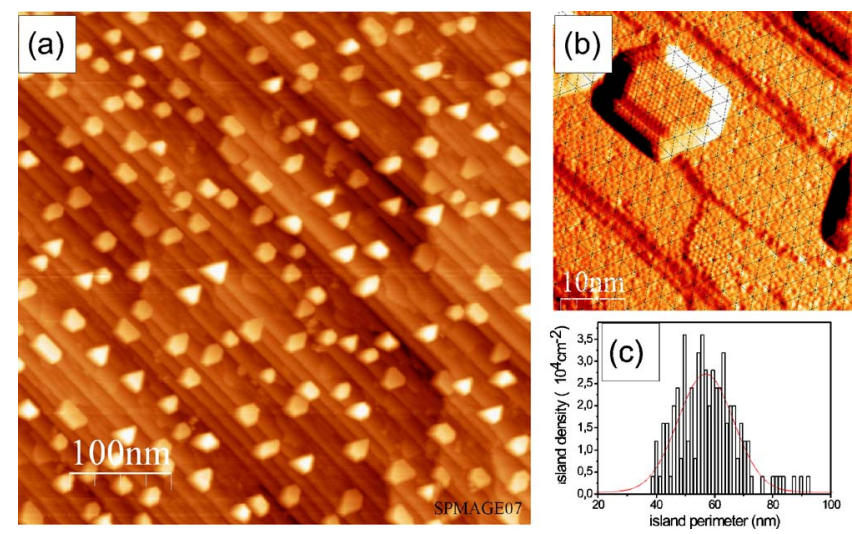

FIG. 2. (Color online) Growth of $0.4 \mathrm{ML}$ of $\mathrm{Co}$ on vicinal $\mathrm{Si}(111)$ at $0.2 \mathrm{ML} / \mathrm{min}$ rate. (a) Empty-state STM topography image $\left(V_{\text {tip }}\right.$ is $\left.+2.1 \mathrm{~V}\right)$ that exhibits a dense array of nanodots over an extended area of the sample. (b) Detailed image of a single island with a hexagonlike geometry with its edges limited by several HUCs of the $\mathrm{Si}(111)-(7 \times 7)$. The flattop and 2 $\times 2$ surface reconstruction are visible. (c) Island perimeter distribution that follows a Gaussian function with a maximum centerd in $57 \mathrm{~nm}$ and a deviation of around $10 \mathrm{~nm}$.

$57 \mathrm{~nm}$ and standard deviation of $10 \mathrm{~nm}$. A two-dimensional fast Fourier transformation of Fig. 2(a) results in well defined ellipses. From the ellipse radii, one can estimate two typical periodicities within the array, either perpendicular $(38.5 \mathrm{~nm})$ or parallel $(72.5 \mathrm{~nm})$ to the surface steps. The perpendicular periodicity corresponds to the average distance between steps in the clean substrate, suggesting that terrace size (miscut angle) can be used to tune the island density at least in one direction. By comparing Figs. 1 and 2, one can observe that the preferred island shape evolves from triangular to hexagonal. The equilibrium shape of the islands is thus closer to the hexagon, i.e., a compact structure that minimizes the island energy while maintaining the original threefold symmetry of the $\mathrm{Si}(111)-(7 \times 7)$ surface. Nonetheless, a careful analysis of Fig. 2(b) reveals that, as depicted in Fig. 1(b), island edges are always contiguous to HUCs of the $\mathrm{Si}(111)-(7 \times 7)$, which thereby delimit the island boundaries also for hexagonal clusters. On the other hand, variation of deposition parameters leads to changes in size and density in the array of quantum dots. Smaller islands but a denser network have been obtained with 1 ML coverage at a $0.3 \mathrm{ML} / \mathrm{min}$ deposition rate, as shown in Fig. 3. In this case, the density of flattop, $\mathrm{CoSi}_{2}$ islands reach the maximum of $3 \times 10^{-3} \mathrm{~nm}^{-2}$, namely, $2 \mathrm{Tbit} / \mathrm{inch}^{2}$.

High surface densities of $\mathrm{CoSi}_{2}$ quantum dots cannot be obtained on flat substrates at the same temperature. Figure 4(a) corresponds to the maximum density of $8 \times 10^{-4} \mathrm{~nm}^{-2}$ that can be achieved by varying the growth conditions. Additionally, RE on flat surfaces is characterized by the presence of a variety of silicide structures, as indicated in Fig. 4(b). Such structures have been analyzed in detail in Ref. 13. Surrounding the $\mathrm{CoSi}_{2}$ quantum dots, we find $\mathrm{Co} \mathrm{RCs}$ and clean $7 \times 7$ patches. However, we also observe a prominent presence of island structures that protrude $4 \AA$ with respect to the flat region, exhibiting flattops with $1 \times 1$ flattop reconstruction and characteristic buckling, as well as $\sqrt{13} \times \sqrt{13}$ reconstructed areas. These two are surface silicide structures with varying Co concentration that are not found during growth on the vicinal surface. The presence of such surface phases points to a less efficient nucleation and growth of $\mathrm{CoSi}_{2}$ in flat $\mathrm{Si}(111)$, as compared to the vicinal surface. 


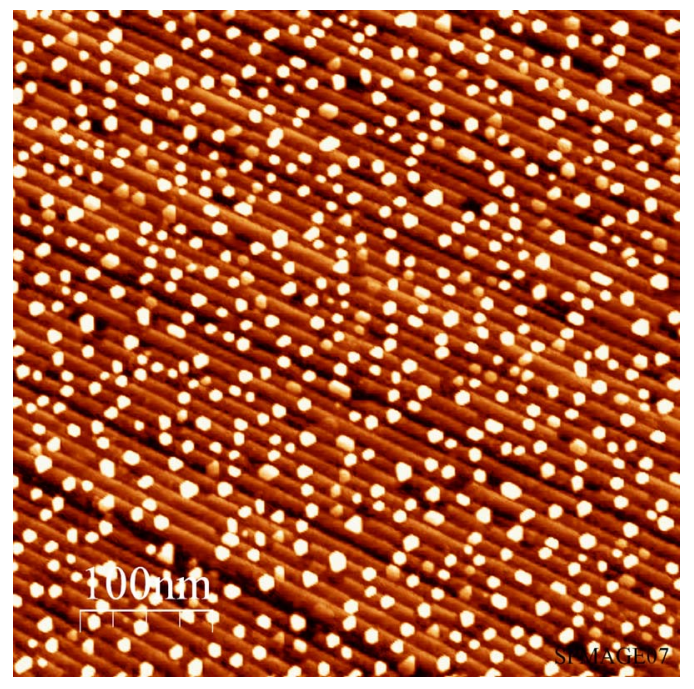

FIG. 3. (Color online) Empty-state STM topography image $\left(V_{\text {tip }}\right.$ is $\left.+2 \mathrm{~V}\right)$ corresponding to the growth of $1 \mathrm{ML}$ of $\mathrm{Co}$ on the vicinal $\mathrm{Si}(111)$ surface at $0.3 \mathrm{ML} / \mathrm{min}$ deposition rate.

Characteristic properties of vicinal surfaces, such as the highly anisotropic diffusion (4-5 times higher diffusion barriers at steps ${ }^{14}$ ) or the discontinuity of the stress field at steps, generally force a high aspect ratio, and hence limit the generation of homogeneous and symmetric islands. ${ }^{15}$ These could be limiting factors in the growth of $\mathrm{CoSi}_{2}$ quantum dots on vicinal surfaces with terraces beyond $250 \AA \AA^{8},{ }^{8}$ but not in terraces below $100 \AA$. In the latter, faster kinetics due to the presence of abundant reactives could lead to a more efficient $\mathrm{CoSi}_{2}$ epitaxy. In fact, step edges are reservoirs of the two-dimensional $\mathrm{Si}$ adatom gas, and hence the density of free $\mathrm{Si}$ atoms on the surface grows linearly with the step
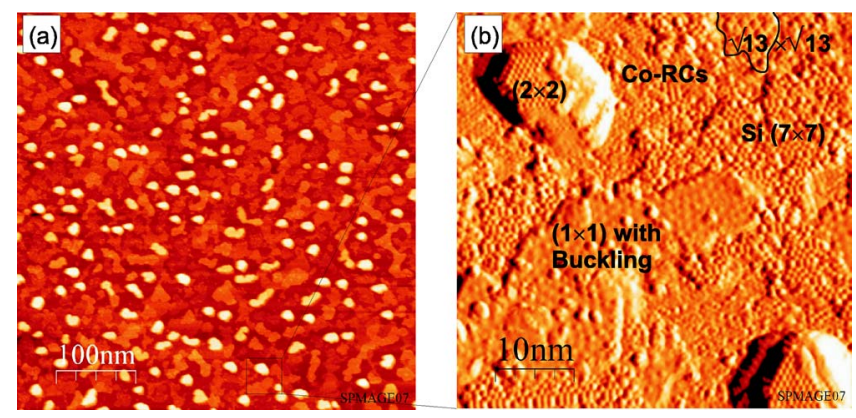

FIG. 4. (Color online) Cobalt silicide surface structures found on flat $\mathrm{Si}(111)$ after 0.4 ML deposition of Co. (a) Empty-state STM topography image $\left(V_{\text {tip }}\right.$ is $\left.+2.1 \mathrm{~V}\right)$ showing complex surface structures with a low density of $\mathrm{CoSi}_{2}$ clusters (bright features). Islands with other geometries and Co concentration coexist. (b) Enlarged STM current image $\left(V_{\text {tip }}\right.$ is $\left.+2.1 \mathrm{~V}\right)$ from (a). Higher $\mathrm{CoSi}_{2}$ islands show $2 \times 2$ surface reconstructions, and appear together with other silicide species, namely, with lower height, $1 \times 1$ reconstructed islands, Co RCs, and $\sqrt{13} \times \sqrt{13}$ patches. density. ${ }^{16}$ Therefore, during deposition by RE, a higher amount of silicon would be available in the immediate vicinity of the steps, compared to the center of the terraces. The enhanced kinetics would explain the absence of less stable surface silicide phases, such as the $1 \times 1$ islands or the $\sqrt{13} \times \sqrt{13}$ structure, which are indeed observed in flat $\operatorname{Si}(111)$.

In conclusion, we have shown that dense self-assembled metallic $\mathrm{CoSi}_{2}$ quantum dot arrays can be grown at relatively low substrate temperatures by RE using a vicinal surface as substrate. The islands are found to grow laterally following the $\operatorname{Si}(111)-(7 \times 7)$ reconstruction and maintaining the threefold symmetry of the substrate surface. A direct comparison with Co deposition on flat $\mathrm{Si}(111)$ reveals the essential role of the steps to enhance the kinetics of the reactive growth process, making it possible to achieve a high density of homogeneous, crystalline $\mathrm{CoSi}_{2}$ islands, while eliminating other undesirable silicide species. The excellent conductive properties of $\mathrm{CoSi}_{2}$ make these metallic quantum dot arrays particularly attractive for nanodevices, such as nanocontacts in molecular electronics.

This work was financed by the Basque Gouvernment (Nanotron Project), the University of the Basque Country (UPV/EHU) (GIU06/27) and the Spanish Ministerio de Educación y Ciencia (MAT2007-63083).

${ }^{1}$ J. E. Ortega and F. J. García de Abajo, Nat. Nanotechnol. 2, 601 (2007). ${ }^{2}$ N. Weiss, T. Cren, M. Epple, S. Rusponi, G. Baudot, S. Rohart, A. Tejeda, V. Repain, S. Rousset, P. Ohresser, F. Scheurer, P. Bencok, and H. Brune, Phys. Rev. Lett. 95, 157204 (2004).

${ }^{3}$ A. Kirakosian, J. L. Mc Chesney, R. Bennewitz, J. N. Crain, J.-L. Lin, and F. J. Himpsel, Surf. Sci. 498, 109 (2002).

${ }^{4}$ R.-L. Vaara, M. Kuzmin, R. E. Perälä, P. Laukkanen, and I. J. Väyrynen, Surf. Sci. 539, 72 (2003).

${ }^{5}$ C. Preinesberger, G. Pruskil, S. K. Becker, M. Dähne, D. V. Vyalikh, S. L. Molodtsov, C. Laubschat, and F. Schiller, Appl. Phys. Lett. 87, 083107 (2005).

${ }^{6}$ S. P. Murarka, Intermetallics 3, 173 (1995).

${ }^{7}$ P. A. Benett, D. G. Cahill, and M. Copel, Phys. Rev. Lett. 73, 452 (1994).

${ }^{8}$ M. A. K. Zilani, H. Xu, X.-S. Wang, and A. T. S. Wee, Appl. Phys. Lett. 88, 023121 (2006).

${ }^{9}$ Z. He, D. J. Smith, and P. A. Bennett, Phys. Rev. Lett. 93, 256102 (2004).

${ }^{10}$ B. Voigtländer and M. Kästner, Phys. Rev. Lett. 81, 858 (1998).

${ }^{11}$ P. A. Bennett, M. Copel, D. G. Cahill, J. Falta, and R. M. Tromp, Phys. Rev. Lett. 69, 1224 (1992).

${ }^{12} \mathrm{We}$ made use of the program wsxm. See I. Horcas, R. Fernández, J. Colchero, J. M. Gómez-Herreo, and A. M. Baró, Rev. Sci. Instrum. 78, 013705 (2007).

${ }^{13}$ M. Löffler, J. Cordón, M. Weinelt, J. E. Ortega, and T. Fauster, Appl. Phys. A: Mater. Sci. Process. 81, 1651 (2005).

${ }^{14} \mathrm{H}$. Ibach, Physics of Surfaces and Interfaces (Springer, Berlin, Heidelberg, New York, 2006).

${ }^{15}$ N. C. Bartelet, R. M. Tromp, and E. D. Williams, Phys. Rev. Lett. 73, 1656 (1994).

${ }^{16}$ R. Tromp, Phys. Rev. Lett. 81, 1050 (1998). 